\title{
Distributed Relay Beamforming in Cognitive Two-Way Networks: SINR Balancing Approach
}

\author{
Seyed Hamid Safavi, Mehrdad Ardebilipour \\ Faculty of Electrical and Computer Engineering, K.N.Toosi University of Technology, Tehran, Iran \\ Email: s.hamid_safavi@ee.kntu.ac.ir,mehrdad@eetd.kntu.ac.ir
}

Received August 16, 2012; revised September 14, 2012; accepted September 24, 2012

\begin{abstract}
In this paper, we study the problem of distributed relay beamforming for a bidirectional cognitive relay network which consists of two secondary transceivers and $K$ ognitive relay nodes and a primary network with a transmitter and receiver, using underlay model. For effective use of spectrum, we propose a Multiple Access Broadcasting (MABC) two-way relaying scheme for cognitive networks. The two transceivers transmit their data towards the relays and then relays retransmit the processed form of signal towards the receiver. Our aim is to design the beamforming coefficients to maximize quality of service (QoS) for the secondary network while satisfying tolerable interference constraint for the primary network. We show that this approach yields a closed-form solution. Our simulation results show that the maximum achievable SINR improved while the tolerable interference temperature becomes not strict for primary receiver.
\end{abstract}

Keywords: Cognitive Radio; Two-Way Relay Networks; Beamforming

\section{Introduction}

With the explosive proliferation of wireless systems, the demand for radio spectrum has been increasing rapidly. As a result, the radio spectrum has become a scarce resource. Cognitive radio (CR) has recently emerged as a promising technology to address the need for intelligent spectrum allocation [1]. In cognitive radio networks, unlicensed (secondary) users can access the licensed (primary) spectrum either on non-interference or interference tolerant basis. There are three main cognitive radio network paradigms: underlay, overlay, and interweave [2]. In the interweave approach, cognitive transmitters are required to sense the spectrum and transmit signals only when frequency holes are available. Spectrum holes are the most obvious opportunities to be exploited by CR, but higher spectrum utilization is anticipated in overlay and underlay approaches where coexistence between the primary user (PU) and secondary users (SUs) is permitted. We have adopted the underlay paradigm due to its advantages from an implementation viewpoint [3] in this work. In the underlay approach, the SUs are allowed to utilize the spectrum of the PU only if the interference generated by the SUs at the primary receivers is below some acceptable threshold which is commonly known as interference temperature [2,4]. This constraint limits the allowed transmit power of SUs and consequently the QoS of the secondary network. To address this issue, cooperation between SUs is a potential way to improve the secondary network QoS while performance of the primary network is not affected. A variety of cooperative strategies have been proposed with different design criteria and assumptions. Among them, distributed beaforming is an efficient technique to enable concurrent transmission of SUs and PUs. Also, in the overlay approach, the SU shares part of its power resources with the PU to provide a relay-assisted transmission.

Recently, motivated by cognitive radio and cooperative communications, cognitive relay networks have gained considerable research interest. As we explained before, distributed relay beamforming, in which the objective is to determine the beamforming weights according to some optimality criterion, have received a lot of attention in non-cognitive relay networks [5-8]. However, the literature on cooperative relaying techniques such as distributed relay beamforming with explicit incorporation of cognitive radio concepts is very sparse. Especially, the use of beamforming in cognitive relay networks is much more challenging because of the existence of the bidirectional interferences between the primary and secondary networks. In addition, two-way relaying technique along with beamforming can further improve the spectrum efficiency in cognitive relay networks $[9,10]$. Two-way relaying scheme can be categorized in three main groups; i.e. two one way relaying, time division broadcasting (TDBC) and multiple access broadcasting (MABC). The MABC approach which is used in this paper, provides a throughput significantly higher than the other approaches 
and well investigated in [11]. A beamforming technique has been proposed in [9] to maximize achievable sum rate in a multi-antenna cognitive two-way relaying network without considering the mutual interference. To the best of our knowledge, the problem of optimal distributed beamforming for an underlay cognitive two-way relay network has not been well addressed.

In this work, we propose a beamforming approach to maximize QoS requirements for the secondary network while satisfying interference temperature constraint for the primary network in an underlay cognitive two-way relay network. Our goal is to obtain beamforming coefficients of the secondary relays as the design parameters, such that the secondary network QoS measured by the signal-to-interference-plus-noise ratio (SINR) at the secondary destination is maximized while interference from secondary network to primary network is constrainted to a predefined value.

Throughout this paper, we use the following standard notations: $(\cdot)^{T}$ and $(\cdot)^{H}$ represent the transpose and the hermitian transpose, respectively. The notation $\operatorname{diag}(\mathbf{A})$ is a vector which contains the diagonal entries of the square matrix $\mathbf{A}$ and $\operatorname{diag}(\mathbf{a})$ is a diagonal matrix whose diagonal elements are different entries of the vector a. $\lambda_{\max }(\mathbf{A})$ and $v_{\max }(\mathbf{A})$ represent principal eigenvalue and eigenvector of matrix $\mathbf{A}$, respectively. $E\{\cdot\}$ denotes the statistical expectation and $\mathbf{I}$ is the identity matrix.

The reminder of paper is organized as follows. In the next section system model and problem formulation for cognitive two-way relaying scheme are described. In Section 3, the SINR balancing under interference constraint is developed. Simulation results are given in Section 4 and finally, the main results are summarized in Section 5.

\section{System Model}

As shown in Figure 1, we consider a set of SUs coexist and operate in the licenced primary band. The secondary



Figure 1. A Two-way cognitive relay network. network consists of a pair of source node exchange information with the assistance of $K$ randomly located relay nodes via MABC two-way relaying scheme. As we consider the underlay paradigm in the model, the secondary network utilizes the primary network's spectrum to transmit its data under a simple two-phase amplify-andphase-adjust-and-forward protocol simultaneously with the primary transmission. It is reasonable for secondary network that have the full channel state information (CSI) by a band manager that interpose between the primary and secondary networks [12].

We denote the channel vector between the $n$ 'th $(n=1,2)$ transceiver and the relays by $\mathbf{f}_{n}=\left[f_{1 n} f_{2 n} \cdots f_{k n}\right]^{T}$ and channel coefficients between the primary transmitter and receiver by $h_{p}$. We also consider mutual interference between the primary and secondary networks in this work. Hence $\mathbf{f}_{p}=\left[f_{1 p} f_{2 p} \cdots f_{k p}\right]^{T}$ denote the interference channel vector from PU transmitter to the relays, while $\mathbf{g}_{p}=\left[g_{1 p} g_{2 p} \cdots g_{k p}\right]^{T}$ is the channel vector between relays and PU receiver. We assume that the forward channels from each transceiver to the relay nodes are reciprocal to the backward channels from the relay nodes to each corresponding transceiver [13]. Also, a flat fading condition is considered so that the channel realizations vary independently from one frame to another while they remain fixed within each frame. Any interference from the secondary transceivers at the primary receiver in the first time slot as well as interference from the primary transmitter at the secondary transceivers in the second time slot is considered as additive white Gaussian noise (AWGN) [4].

During the first time slot (multiple access phase), both transceivers simultaneously transmit their data to the relays. The received signals in relays from transceivers as well as interference from PU transmitter can be represented, in vector form, as

$$
\mathbf{x}=\sqrt{P_{1}} \mathbf{f}_{1} s_{1}+\sqrt{P_{2}} \mathbf{f}_{2} s_{2}+\sqrt{P_{p}} \mathbf{f}_{p} s_{p}^{(1)}+\mathbf{v}
$$

where $\mathbf{x}$ is the $K \times 1$ complex vector of the received signals at the relays, $P_{1}, P_{2}$ and $P_{p}$ are the transmit powers of Transceivers 1, 2 and PU transmitter, respectively. Let $S_{1}, S_{2}$ represent the information symbols transmitted by transceivers 1,2 and $s_{p}^{(1)}, s_{p}^{(2)}$ represent the information symbols transmitted by PU transmitter in the first and second time slot respectively. $v$ is the $K \times 1$ complex vector of the relay noises with covariance matrix $\sigma^{2} \mathbf{I}$.

In the second time slot (broadcasting phase), the $i$ 'th relay multiplies its received signal by a complex weight $w_{i}^{*}$ and transmits the so-obtained signal can be expressed as

$$
\mathbf{t}=\mathbf{W} \mathbf{x}
$$

where $\mathbf{W}=\left[w_{1}^{*}, w_{2}^{*}, \cdots, w_{k}^{*}\right]$. The received signal in two 
transceivers can be written as:

$$
\begin{aligned}
y_{1} & =\mathbf{f}_{1}^{T} \mathbf{W} \mathbf{x}+n_{1} \\
& =\mathbf{f}_{1}^{T} \mathbf{W}\left(\sqrt{P_{1}} \mathbf{f}_{1} s_{1}+\sqrt{P_{2}} \mathbf{f}_{2} s_{2}+\sqrt{P_{p}} \mathbf{f}_{p} s_{p}^{(1)}+\mathbf{v}\right)+n_{1} \\
y_{2} & =\mathbf{f}_{2}^{T} \mathbf{W} \mathbf{x}+n_{2} \\
& =\mathbf{f}_{2}^{T} \mathbf{W}\left(\sqrt{P_{1}} \mathbf{f}_{1} s_{1}+\sqrt{P_{2}} \mathbf{f}_{2} s_{2}+\sqrt{P_{p}} \mathbf{f}_{p} s_{p}^{(1)}+\mathbf{v}\right)+n_{2}
\end{aligned}
$$

Using $\mathbf{a}^{T} \operatorname{diag}(\mathbf{b})=\mathbf{b}^{T} \operatorname{diag}(\mathbf{a})$, we rewrite (3) and (4) as

$$
\begin{aligned}
y_{1} & =\sqrt{P_{1}} \mathbf{w}^{H} \mathbf{F}_{1} \mathbf{f}_{1} s_{1}+\sqrt{P_{2}} \mathbf{w}^{H} \mathbf{F}_{1} \mathbf{f}_{2} s_{2} \\
& +\sqrt{P_{p}} \mathbf{w}^{H} \mathbf{F}_{1} \mathbf{f}_{p} s_{p}^{(1)}+\mathbf{w}^{H} \mathbf{F}_{1} \mathbf{v}+n_{1} \\
y_{2} & =\sqrt{P_{1}} \mathbf{w}^{H} \mathbf{F}_{2} \mathbf{f}_{1} s_{1}+\sqrt{P_{2}} \mathbf{w}^{H} \mathbf{F}_{2} \mathbf{f}_{2} s_{2} \\
& +\sqrt{P_{p}} \mathbf{w}^{H} \mathbf{F}_{2} \mathbf{f}_{p} s_{p}^{(1)}+\mathbf{w}^{H} \mathbf{F}_{2} \mathbf{v}+n_{2}
\end{aligned}
$$

where $\mathbf{w} \triangleq \operatorname{diag}\left(\mathbf{W}^{H}\right), \quad \mathbf{F}_{1} \triangleq \operatorname{diag}\left(\mathbf{f}_{1}\right), \mathbf{F}_{2} \triangleq \operatorname{diag}\left(\mathbf{f}_{2}\right)$. The noise process is assumed to be zero-mean and spatially white with variance $\boldsymbol{\sigma}^{2}$. We will later explain how each relay can compute its own optimal beamforming weight. Since the knowledge of $\mathbf{f}_{1}$ and $s_{1}$ are available at Transceiver 1, thus transceiver 1 can subtracts the first term in (5) and manipulate the remaining term to have

$$
\begin{aligned}
\tilde{y}_{1} & \triangleq y_{1}-\sqrt{P_{1}} \mathbf{w}^{H} \mathbf{F}_{1} \mathbf{f}_{1} s_{1} \\
& =\underbrace{\sqrt{P_{2}} \mathbf{w}^{H} \mathbf{F}_{1} \mathbf{f}_{2} s_{2}}_{\text {desired signal }}+\underbrace{\sqrt{P_{p}} \mathbf{w}^{H} \mathbf{F}_{1} \mathbf{f}_{p} s_{p}^{(1)}}_{\text {interference }}+\underbrace{\mathbf{w}^{H} \mathbf{F}_{1} \mathbf{v}+n_{1}}_{\text {noise }}
\end{aligned}
$$

and similarly

$$
\begin{aligned}
\tilde{y}_{2} & \triangleq y_{2}-\sqrt{P_{2}} \mathbf{w}^{H} \mathbf{F}_{2} \mathbf{f}_{2} s_{2} \\
& =\underbrace{\sqrt{P_{1}} \mathbf{w}^{H} \mathbf{F}_{2} \mathbf{f}_{1} s_{1}}_{\text {desired signal }}+\underbrace{\sqrt{P_{p}} \mathbf{w}^{H} \mathbf{F}_{2} \mathbf{f}_{p} s_{p}^{(1)}}_{\text {interference }}+\underbrace{\mathbf{w}^{H} \mathbf{F}_{2} \mathbf{v}+n_{2}}_{\text {noise }}
\end{aligned}
$$

The received signal at the primary receiver can be expressed as

$$
\begin{aligned}
y_{p} & =\sqrt{P_{p}} h_{p} s_{p}^{(2)}+\mathbf{g}_{p}^{T} \mathbf{W} \mathbf{x}+n_{p} \\
& =\sqrt{P_{p}} h_{p} s_{p}^{(2)}+\mathbf{g}_{p}^{T} \mathbf{W} \\
& \times\left(\sqrt{P_{1}} \mathbf{f}_{1} s_{1}+\sqrt{P_{2}} \mathbf{f}_{2} s_{2}+\sqrt{P_{p}} \mathbf{f}_{p} s_{p}^{(1)}+\mathbf{v}\right)+n_{p} \\
& =\underbrace{\sqrt{P_{p}} h_{p} s_{p}^{(2)}}_{\text {desiredsignal }}+\underbrace{n_{p}}_{\text {noise }} \\
& +\underbrace{\sqrt{P_{1}} \mathbf{w}^{H} \mathbf{G}_{p} \mathbf{f}_{1} s_{1}+\sqrt{P_{2}} \mathbf{w}^{H} \mathbf{G}_{p} \mathbf{f}_{2} s_{2}+\mathbf{w}^{H} \mathbf{G}_{p} \mathbf{v}}_{\text {interferencefrom secondary network }} \\
& +\underbrace{\sqrt{P_{p}} \mathbf{w}^{H} \mathbf{G}_{p} \mathbf{f}_{p} s_{p}^{(1)}}_{\text {self interference }}
\end{aligned}
$$

\section{SINR Balancing}

In this section, our goal is to find the beamforming weight vector $\mathbf{W}$ in order to SINR balancing at the secondary network subject to an interference power constraint at the primary network. Mathematically, the optimization problem can be represented as follows

$$
\begin{aligned}
& \max _{\mathbf{w}} \min \left(\operatorname{SINR}_{1}, \operatorname{SINR}_{2}\right) \\
& \text { Subject to } \quad P_{I} \leq I_{\text {th }}
\end{aligned}
$$

where $S I N R_{m}$ is defined as the ratio of the desired signal power to the interference plus noise power at the $m$ 'th transceiver for $m=1,2$ and $P_{I}$ denotes the interference power. These parameters can be calculated as follows

$$
\begin{aligned}
& \operatorname{SINR}_{m}=\frac{P_{s_{m}}}{P_{i_{m}}+P_{n_{m}}} m=1,2 \\
& P_{s_{1}}=E\left\{P_{2} \mathbf{w}^{H} \mathbf{F}_{1} \mathbf{f}_{2} S_{2} S_{2}^{*} \mathbf{f}_{2}^{H} \mathbf{F}_{1}^{H} \mathbf{w}\right\} \\
& =P_{2} E\left\{\mathbf{w}^{H} \mathbf{F}_{1} \mathbf{f}_{2} \mathbf{f}_{2}^{H} \mathbf{F}_{1}^{H} \mathbf{w}\right\} \underbrace{E\left\{\left|S_{2}\right|^{2}\right\}}_{=1} \\
& =P_{2} \mathbf{w}^{H} \mathbf{h} \mathbf{h}^{H} \mathbf{w} \\
& =P_{2} \mathbf{w}^{H} \mathbf{A} \mathbf{w}
\end{aligned}
$$

where $\mathbf{h} \triangleq \mathbf{F}_{1} \mathbf{f}_{2}=\mathbf{F}_{2} \mathbf{f}_{1}, \quad \mathbf{A} \triangleq \mathbf{h h}^{H}$.

$$
\begin{aligned}
P_{i_{1}} & =E\left\{P_{2} \mathbf{w}^{H} \mathbf{F}_{1} \mathbf{f}_{p} s_{p}^{(1)} s_{p}^{(1)^{*}} \mathbf{f}_{p}^{H} \mathbf{F}_{1}^{H} \mathbf{w}\right\} \\
& =P_{p} E\left\{\mathbf{w}^{H} \mathbf{F}_{1} \mathbf{f}_{p} \mathbf{f}_{p}^{H} \mathbf{F}_{1}^{H} \mathbf{w}\right\} \underbrace{E\left\{\left|s_{p}^{(1)}\right|^{2}\right\}}_{=1} \\
& =P_{2} \mathbf{w}^{H} \mathbf{k} \mathbf{k}^{H} \mathbf{w}
\end{aligned}
$$

where $\mathbf{K} \triangleq \mathbf{F}_{1} \mathbf{f}_{p}$.

$$
\begin{aligned}
P_{n_{1}} & =E\left\{\mathbf{w}^{H} \mathbf{F}_{1} \boldsymbol{v} \boldsymbol{v}^{H} \mathbf{F}_{1}^{H} \mathbf{w}\right\}+E\left\{\left|n_{1}\right|^{2}\right\} \\
& =\mathbf{w}^{H} \mathbf{F}_{1} E\left\{\boldsymbol{v} \boldsymbol{v}^{H}\right\} \mathbf{F}_{1}^{H} \mathbf{w}+\sigma^{2} \\
& =\sigma^{2} \mathbf{w}^{H} \mathbf{D}_{1} \mathbf{w}+\sigma^{2}
\end{aligned}
$$

where $\mathbf{D}_{1} \triangleq \mathbf{F}_{1} \mathbf{F}_{1}^{H}$, similarly

$$
\left\{\begin{array}{l}
P_{s_{2}}=P_{1} \mathbf{w}^{H} \mathbf{A} \mathbf{w} \\
P_{i_{2}}=P_{p} \mathbf{w}^{H} \mathbf{L} \mathbf{L}^{H} \mathbf{w} \\
P_{n_{2}}=\sigma^{2} \mathbf{w}^{H} \mathbf{D}_{2} \mathbf{w}+\sigma^{2}
\end{array}\right.
$$

where $\mathbf{L} \triangleq \mathbf{F}_{2} \mathbf{f}_{p}, \quad \mathbf{D}_{2} \triangleq \mathbf{F}_{2} \mathbf{F}_{2}^{H}$.

$$
\begin{aligned}
\operatorname{SINR}_{1} & =\frac{P_{2} \mathbf{w}^{H} \mathbf{A} \mathbf{w}}{\sigma^{2}+\sigma^{2} \mathbf{w}^{H} \mathbf{D}_{1} \mathbf{w}+P_{p} \mathbf{w}^{H} \mathbf{k} \mathbf{k}^{H} \mathbf{w}} \\
& =\frac{P_{2} \mathbf{w}^{H} \mathbf{A} \mathbf{w}}{\sigma^{2}+\mathbf{w}^{H}\left(\sigma^{2} \mathbf{D}_{1}+P_{p} \mathbf{k} \mathbf{k}^{H}\right) \mathbf{w}} \\
& =\frac{P_{2} \mathbf{w}^{H} \mathbf{A} \mathbf{w}}{\sigma^{2}+\mathbf{w}^{H} \mathbf{B} \mathbf{w}}
\end{aligned}
$$

and similarly 


$$
\begin{aligned}
\operatorname{SINR}_{1} & =\frac{P_{1} \mathbf{w}^{H} \mathbf{A} \mathbf{w}}{\sigma^{2}+\sigma^{2} \mathbf{w}^{H} \mathbf{D}_{2} \mathbf{w}+P_{p} \mathbf{w}^{H} \mathbf{L} \mathbf{L}^{H} \mathbf{w}} \\
& =\frac{P_{1} \mathbf{w}^{H} \mathbf{A} \mathbf{w}}{\sigma^{2}+\mathbf{w}^{H}\left(\sigma^{2} \mathbf{D}_{2}+P_{p} \mathbf{L} \mathbf{L}^{H}\right) \mathbf{w}} \\
& =\frac{P_{1} \mathbf{w}^{H} \mathbf{A} \mathbf{w}}{\sigma^{2}+\mathbf{w}^{H} \mathbf{C} \mathbf{w}}
\end{aligned}
$$

where

$$
\left\{\begin{array}{l}
\mathbf{B}=\sigma^{2} \mathbf{D}_{1}+P_{p} \mathbf{k} \mathbf{k}^{H} \\
\mathbf{C}=\sigma^{2} \mathbf{D}_{2}+P_{p} \mathbf{L} \mathbf{L}^{H}
\end{array}\right.
$$

Using (9) so the interference component power which consists of secondary network interference and self interference can be written as

$$
\begin{aligned}
& P_{I} \triangleq E\left\{P_{1} \mathbf{w}^{H} \mathbf{G}_{p} \mathbf{f}_{1} s_{1} s_{1}^{*} \mathbf{f}_{1}^{H} \mathbf{G}_{p}^{H} \mathbf{w}\right\} \\
& +E\left\{P_{2} \mathbf{w}^{H} \mathbf{G}_{p} \mathbf{f}_{2} s_{2} s_{2}^{*} \mathbf{f}_{2}^{H} \mathbf{G}_{p}^{H} \mathbf{w}\right\} \\
& +E\left\{\mathbf{w}^{H} \mathbf{G}_{p} \mathbf{v} \mathbf{v}^{H} \mathbf{G}_{p}^{H} \mathbf{w}\right\} \\
& +E\left\{P_{2} \mathbf{w}^{H} \mathbf{G}_{p} \mathbf{f}_{p} s_{p}^{(1)} s_{p}^{(1)} \mathbf{f}_{2}^{H} \mathbf{G}_{p}^{H} \mathbf{w}\right\} \\
& =P_{1} \mathbf{w}^{H} \mathbf{U} \mathbf{U}^{H} \mathbf{w} \underbrace{E\left\{\left|s_{1}\right|^{2}\right\}}_{=1}+P_{2} \mathbf{w}^{H} \mathbf{Y} \mathbf{Y}^{H} \mathbf{w} \underbrace{E\left\{\left|s_{2}\right|^{2}\right\}}_{=1} \\
& +P_{p} \mathbf{w}^{H} \mathbf{Z} \mathbf{Z}^{H} \mathbf{w} \underbrace{E\left\{\left|s_{p}^{(1)}\right|^{2}\right\}}_{=1}+\mathbf{w}^{H} \mathbf{G}_{p} \underbrace{E\left\{\mathbf{v} \boldsymbol{v}^{H}\right\}}_{\sigma^{2}} \mathbf{G}_{p}^{H} \mathbf{w} \\
& =\mathbf{w}^{H} \mathbf{Q} \mathbf{w}
\end{aligned}
$$

where

$$
\left\{\begin{array}{l}
\mathbf{U}=\mathbf{G}_{p} \mathbf{f}_{1} \\
\mathbf{Y}=\mathbf{G}_{p} \mathbf{f}_{2} \\
\mathbf{Z}=\mathbf{G}_{p} \mathbf{f}_{p}
\end{array}\right.
$$

and

$$
\mathbf{Q}=P_{1} \mathbf{U} \mathbf{U}^{H}+P_{2} \mathbf{Y} \mathbf{Y}^{H}+P_{p} \mathbf{Z} \mathbf{Z}^{H}+\sigma^{2} \mathbf{G}_{p} \mathbf{G}_{p}^{H}
$$

Note that at the optimum, it is required that

$$
S I N R_{1}=S I N R_{2}
$$

Otherwise, if, for example, $\mathrm{SINR}_{1}>\mathrm{SINR}_{2}$, then $P_{2}$ can be reduced such that $\mathrm{SINR}_{1}=\mathrm{SINR}_{2}$ and this reduction of $P_{2}$ will not violate the power constraint. Using (15) and (19) the optimization problem (10) can be written as

$$
\begin{array}{ll}
\max & \frac{P_{2} \mathbf{w}^{H} \mathbf{A w}}{\sigma^{2}+\mathbf{w}^{H} \mathbf{B w}} \\
\text { S.t. } & \mathbf{w}^{H} \mathbf{Q w} \leq I_{\text {th }}
\end{array}
$$

to solve (20), let us write the weight vector $\mathbf{w}$ as

$$
\left\{\begin{array}{l}
\mathbf{W}=\sqrt{I_{t h}} \mathbf{Q}^{-\frac{1}{2}} \tilde{\mathbf{w}} \\
\tilde{\mathbf{w}}^{H} \tilde{\mathbf{w}}=1
\end{array}\right.
$$

then we can rewrite the optimization problem as

$$
\begin{array}{ll}
\max & \frac{P_{2} I \tilde{\mathbf{w}}^{H} \tilde{\mathbf{A}} \tilde{\mathbf{w}}}{\sigma^{2}+I \tilde{\mathbf{w}}^{H} \tilde{\mathbf{B}} \tilde{\mathbf{w}}} \\
\text { S.t. } & \|\tilde{\mathbf{w}}\|^{2}=1, I \leq I_{t h}
\end{array}
$$

It is easy to show that the inequality constraint in (22) will be satisfied with equality at the optimum. As the objective function in (22) is monotonically increasing in $\boldsymbol{I}$, for any value of $\mathbf{w}$, this objective function is maximized for $I-I_{t h}$.

$$
\begin{array}{ll}
\max & \frac{P_{2} I_{t h} \tilde{\mathbf{w}}^{H} \tilde{\mathbf{A}} \tilde{\mathbf{w}}}{\sigma^{2}+I_{t h} \tilde{\mathbf{w}}^{H} \tilde{\mathbf{B}} \tilde{\mathbf{w}}} \\
\text { S.t. } & \|\tilde{\mathbf{w}}\|^{2}=1
\end{array}
$$

It is obvious that the optimization problem (23) is in the form of Rayleigh-Ritz ratio, in which objective function is globally maximized when $\boldsymbol{W}^{\boldsymbol{H}}$ chosen as the constant factor of the principal eigenvector of the matrix $\left(I_{t h} \tilde{\mathbf{B}}+\sigma^{2} \mathbf{I}\right)^{-1} \tilde{\mathbf{A}}$.

$$
\tilde{\mathbf{w}}=v_{\max }\left\{\left(I_{t h} \tilde{\mathbf{B}}+\sigma^{2} \mathbf{I}\right)^{-1} \tilde{\mathbf{A}}\right\}
$$

as a result, the beamforming weight vector can be written as

$$
\begin{aligned}
& \mathbf{w}=\sqrt{I_{t h}} \mathbf{Q}^{-1 / 2} v_{\max } \\
& \cdot\left\{\left(I_{t h} \mathbf{Q}^{-1 / 2} \mathbf{B} \mathbf{Q}^{-1 / 2}+\sigma^{2} \mathbf{I}\right)^{-1} \mathbf{Q}^{-1 / 2} \mathbf{A} \mathbf{Q}^{-1 / 2}\right\}
\end{aligned}
$$

and the maximum achievable SINR can be expressed as

$$
\begin{aligned}
& \operatorname{SINR}_{\max }=P_{2} I_{\text {th }} \\
& \times \lambda_{\max }\left\{\left(I_{t h} \mathbf{Q}^{-1 / 2} \mathbf{B} \mathbf{Q}^{-1 / 2}+\sigma^{2} \mathbf{I}\right)^{-1} \mathbf{Q}^{-1 / 2} \mathbf{A} \mathbf{Q}^{-1 / 2}\right\}
\end{aligned}
$$

As the level of interference temperature can be estimated at the secondary network [2] and we assume that the secondary network have full CSI, optimal beamforming coefficient in each relay can be calculated from (25).

\section{Simulation Results}

In our simulation results we consider a secondary network with $K=20,30$, 40 relay nodes, and the channel coefficients are generated independently as complex Gaussian random variables with unit variance in each simulation run. All noise powers including relay noises, secondary and primary receiver noises is assumed to be 0 dBW. Throughout our numerical examples, the transmit power of transceivers and PU is also considered to be equal to $0 \mathrm{dBW}$. The average value of each quantity is obtained by averaging the corresponding quantity over $10^{4}$ simulation runs.

Figure 2 illustrates the average values of the maxi- 
mum achievable SINRs versus the maximum interference power that primary receiver can tolerate for three different values of $K$. As can be seen from this figure, as we increase $K$, the maximum achievable SINRs increase. The achieved improvement from 30 relays to 40 has become lower than the improvement of 20 to 30.

Figure 3 shows the average values of the relay transmit power for three different values of $K$. It is reasonable that, as we increase the number of relays, total power dissipated in the relays doesn't change considerably for fixed tolerable interference. However because of the beamforming effect and phase compensation, SINR of each transceiver's is improved.

Figure 4 illustrates the average values of the maximum achievable SINRs versus the maximum interference power that primary receiver can tolerate for 30 relays and two different scenarios: 1) $\sigma_{f_{1}}^{2}=\sigma_{f_{2}}^{2}=0 \mathrm{~dB}$ and 2) $\sigma_{f_{1}}^{2}=\sigma_{f_{2}}^{2}=3 \mathrm{~dB}$. As can be seen from this figure,

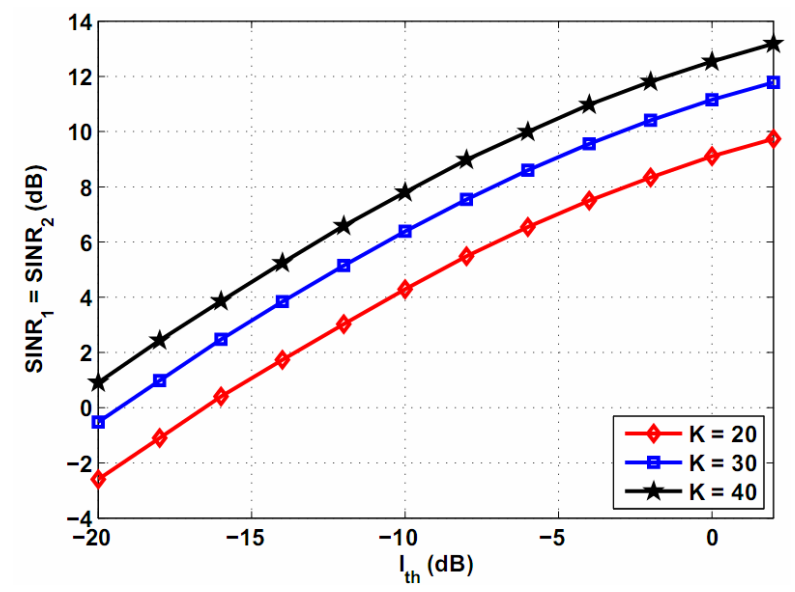

Figure 2. The average values of the maximum achievable SINRs versus the interference temperature for three different values of $K$.



Figure 3. Total relay Power dissipated in the network versus the interference temperature for three different values of $K$.

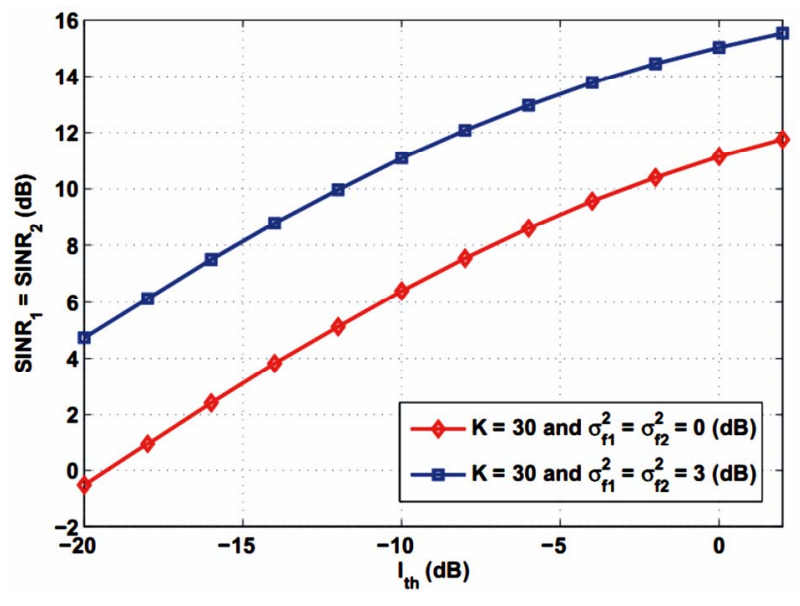

Figure 4. The average values of the maximum achievable SINRs versus the interference temperature for two different scenarios; 1) $\sigma_{f_{1}}^{2}=\sigma_{f_{2}}^{2}=0 \mathrm{~dB}$;2) $\sigma_{f_{1}}^{2}=\sigma_{f_{2}}^{2}=3 \mathrm{~dB}$.

by increasing the interference temperature and improving the quality of secondary channels, SINR improvement decreases. Because the interference constraint become strict.

\section{Conclusion}

In this paper, we developed the distributed relay beamforming for an underlay bidirectional cognitive network which consists of two transceivers and $K$ relay nodes between them all equipped with single-antenna in the presence of primary network. For effective use of spectrum, MABC two-way relaying which needs two time slots to swap two symbols between the two transceivers proposed for cognitive networks. We study SINR balancing technique where the smaller of the two transceiver SINRs is maximized while keeping the interference power below interference temperature. We herein have shown that this approach leads to a closed-form solution.

\section{REFERENCES}

[1] S. Haykin, "Cognitive Radio: Brain-Empowered Wireless Communications," IEEE Journal on Selected Areas in Communications, Vol. 23, No. 2, 2005, pp. 201-220. doi:10.1109/JSAC.2004.839380

[2] A. Goldsmith, S. A. Jafar, I. Maric and S. Srinivasa, "Breaking Spectrum Gridlock with Cognitive Radios: An Information Theoretic Perspective," Proceedings of the IEEE, Vol. 97, No. 5, 2009, pp. 894-914. doi:10.1109/JPROC.2009.2015717

[3] R. Zhang, “On Peak versus Average Interference Power Constraints for Protecting Primary Users in Cognitive Radio Networks,” IEEE Transactions on Wireless Communications, Vol. 8, No. 4, 2009, pp. 2112-2120. doi:10.1109/TWC.2009.080714

[4] L. Musavian, S. Aissa and S. Lambotharan, "Effective Capacity for Interference and Delay Constrained Cogni- 
tive Radio Relay Channels,” IEEE Transactions on Wireless Communications, Vol. 9, No. 5, 2010, pp. 1698-1707. doi:10.1109/TCOMM.2010.05.090600

[5] V. Havary-Nassab, S. Shahbazpanahi, A. Grami and Z.-Q. Luo, "Distributed Beamforming for Relay Networks Based on Second-Order Statistics of the Channel State Information," IEEE Transactions on Signal Processing, Vol. 56, No. 9, 2008, pp. 4306-4316. doi:10.1109/TSP.2008.925945

[6] S. Fazeli-Dehkordy, S. Shahbazpanahi and S. Gazor, "Multiple Peer-to-Peer Communications Using a Network of Relays," IEEE Transactions on Signal Processing, Vol. 57, No. 8, 2009, pp. 3053-3062.

[7] J. Li, A. P. Petropulu and H. V. Poor, "Cooperative Transmission for Relay Networks Based on Second-Order Statistics of Channel State Information," IEEE Transactions on Signal Processing, Vol. 59, No. 3, 2011, pp. 1280-1291. doi:10.1109/TSP.2010.2094614

[8] A. S. Behbahani and A. M. Eltawil, "Amplify and Forward Relay Networks under Intereference Power Constraint," IEEE Transactions on Wireless Communications, Vol. 8, No. 11, 2009, pp. 5422-5426. doi:10.1109/TWC.2009.081522
[9] K. Jitvanichphaibool, Y.-C. Liang and R. Zhang, "Beamforming and Power Control for Multi-Antenna Cognitive Two-Way Relaying," IEEE Wireless Communications and Networking Conference, Singapore, 5-8 April 2009, pp. 1-6. doi:10.1109/WCNC.2009.4917611

[10] A. Alizadeh, S. M. S. Sadough and N. Khajavi, "Optimal Beamforming in Cognitive Two-Way Relay Networks," IEEE 21st International Symposium on Personal Indoor and Mobile Radio Communications (PIMRC), Instanbul, 26-29 September 2010, p. 2331.

[11] V. Havary-Nassab, S. Shahbazpanahi and A. Grami, “Optimal Distributed Beamforming for Two-Way Relay Networks," IEEE Transactions on Signal Processing, Vol. 58, No. 3, 2010, pp. 1238-1250. doi:10.1109/TSP.2009.2026067

[12] J. M. Peha, "Approaches to Spectrum Sharing," IEEE Communications Magazine, Vol. 43, No. 2, 2005, pp. 10 12. doi:10.1109/MCOM.2005.1391490

[13] M. Zeng, R. Zhang and S. Cui, “On Design of Collaborative Beamforming for Two-Way Relay Networks,” IEEE Transactions on Signal Processing, Vol. 59, No. 5, 2011 pp. 2284-2295. doi:10.1109/TSP.2011.2107906 